\title{
Congrès 2004 de l'American Urological Association San Francisco, Californie, (8-13 Mai 2004)
}

\author{
Wayne J. HELLSTROM ${ }^{1}$, Jacques BUVAT ${ }^{2}$ \\ ${ }^{1}$ New Orleans, Louisiana, USA ${ }^{2}$ Lille, France
}

Le congrès 2004 de l'American Urological Association s'est tenu à San Francisco, et a connu comme chaque année, une fréquentation massive. Comme l'année précédente à Chicago, une place importante a été réservée aux aspects cliniques des problèmes sexuels, et plus encore à la recherche fondamentale en matière de sexualité. Avec tout particulièrement une "podium session " de 2 heures réservée à des communications orales, et 3 séances de posters suivies de discussion. A ceci s'ajoutant de nombreuses mises au point et cours consacrés aux mêmes domaines.

Le Pr Wayne Hellstrom, de la Tulane University de la Nouvelle Orleans, président de la Société de Médecine Sexuelle Nord-Américaine (SMSNA), chapitre nord-américain de I'ISSIR, nous a fait I'honneur de nous confier l'enregistrement de son " take-home message ", consacré aux aspects sexuels des présentations du congrès, et qui en reprend toutes les présentations marquantes. Nous l'en remercions et vous en trouverez ci-après la traduction remise en forme et assortie de quelques commentaires personnels (JB).

\section{Introduction}

Depuis la prise de conscience de la grande fréquence des dysfonctions érectiles (DE) dans la population générale, et la mise au point de traitements efficaces, le champ de la médecine sexuelle s'est esquissé, et tend aujourd'hui à se préciser et s'élargir.

\section{Epidémiologie}

Afin de mieux préciser la place des DE dans la pratique urologique générale, plus de 100 urologues français ont participé à une enquête dans laquelle ils ont inclus chacun 10 patients consécutifs, quel que soit le motif de leur consultation [1]. Parmi ces 1303 patients (âge moyen 57,2 $\pm 14,5$ ans), le score moyen de l'lleF-5 était de respectivement $13,0 \pm 5,3$ et de $22,6 \pm 4,0$ chez les hommes avec $(n=818)$ et sans $(n=485) D E$. Les auteurs ont trouvé une prévalence de la DE beaucoup plus élevée en pratique urologique qu'elle ne l'est en médecine générale. La sévérité de la DE augmentait avec l'âge, mais les patients de plus de 70 ans en étaient moins gênés, suggérant qu'il existe une diminution des attentes sexuelles avec l'âge. Les auteurs ont recommandé que les normes de l'IIEF-5 soient ajustées à l'âge, pour une utilisation plus fiable chez le sujet âgé.

\section{Cancer de la Prostate}

La technique de préservation nerveuse, susceptible de préserver la fonction érectile, a fait beaucoup pour que la prostatectomie radicale rétropubienne soit aujourd'hui le traitement le plus utilisé aux USA en cas de cancer localisé de la prostate. Récemment, l'interposition du nerf sural a également été préconisée pour préserver la puissance des hommes subissant une prostatectomie radicale sans préservation nerveuse.

Dans une étude allemande, une prostatectomie radicale avec préservation nerveuse a été pratiquée chez 688 hommes, dont $82 \%$ ont complété tous les questionnaires de $D E$, et 432 avaient une fonction érectile pré-opératoire normale [2]. L'analyse finale a inclus 138 hommes avec préservation nerveuse unilatérale, 273 hommes avec préservation bilatérale, et 21 hommes avec préservation unilatérale et interposition controlatérale du nerf sural. Dans ce dernier groupe (interposition du nerf sural), la fonction érectile post-opératoire ne fut pas significativement différente de celle du groupe avec préservation nerveuse unilatérale seule, mais fut, comme on pouvait s'y attendre, significativement plus mauvaise qu'en cas de préservation nerveuse bilatérale. A ce stade, ces auteurs concluent que la greffe du nerf sural reste une méthode expérimentale, et nécessite des études à plus long terme.

\section{Correspondance :}

Dr Jacques BUVAT - Email jacques-buvat@wanadoo.fr 
Lors du dernier congres de l'AUA, Padma-Nathan et al. avaient rapporté une amélioration à long terme de la fonction érectile spontanée chez 76 hommes traités par prise vespérale de sildénafil pendant 9 mois après prostatectomie radicale rétropubienne avec conservation nerveuse. Cette année, le même groupe a rapporté les résultats à long terme du monitoring des érections par le Rigiscan dans le sous-groupe des 54 hommes de cette étude qui avaient une fonction érectile pré-opératoire normale, selon un score $\geq 8$ pour la somme des questions Q3 et Q4 de l'IIEF, et des résultats normaux au Rigiscan (plus de 10 minutes consécutives de rigidité $>55 \%$ à la base) [3]. Les patients avaient reçu de façon randomisée pendant 7 mois soit $50 \mathrm{mg}$, soit $100 \mathrm{mg}$ de Sildenafil, soit un placebo, chaque soir, commencés 4 semaines après l'intervention. Deux nuits consécutives ont été enregistrées par le Rigiscan $4,12,24,36$ et 48 semaines après l'opération.

Dix des 35 hommes traités par le sildénafil (29\%) et un seul des 19 hommes traités par placebo (5\%) avaient retrouvé des possibilités de rapports spontanés un mois après l'arrêt de tout traitement. Avant l'opération il n'y avait pas de différence significative entre les sous-groupes en ce qui concerne le nombre de minutes avec rigidité de plus de $55 \%$ à la base ou à l'extrémité du pénis. Quarante huit semaines après l'intervention, la mesure la plus discriminante entre répondeurs et non-répondeurs fut la durée de la rigidité à plus de $55 \%$ à l'extrémité du pénis, cette durée étant plus longue dans le sous-groupe ayant reçu $100 \mathrm{mg}$ de sildénafil. On peut donc supposer qu'une inhibition chronique de la phosphodiesterase de type 5 (PDE-5) améliore l'oxygénation du tissu caverneux par l'intermédiaire d'une facilitation des érections nocturnes, et/ou induit une régénération neuronale.

\section{Traitement oral des DE}

Le traitement oral est devenu le traitement de première intention pour la majorité des hommes souffrant de DE. On pense par ailleurs aujourd'hui que les érections nocturnes contribuent au maintien de l'intégrité morphologique et dynamique des fibres musculaires lisses du corps caverneux. On sait que l'administration de sildenafil au coucher peut augmenter ces érections nocturnes. Pour ces raisons, une équipe allemande a étudié les effets à long terme du sildénafil [4]. Dans une étude prospective et randomisée avec groupe témoin, 76 hommes d'âge moyen 47 ans présentant une DE depuis plus de 6 mois ont reçu $50 \mathrm{mg}$ de sildénafil chaque soir, ou 50 à $100 \mathrm{mg}$ de sildénafil à la demande, pendant un an. Un troisième groupe n'a reçu aucun traitement.

Après arrêt de tout traitement pendant un mois, $59 \%$ des hommes du groupe prenant le sildénafil chaque soir, et 10 $\%$ du groupe prenant le traitement à la demande, avaient un score du domaine de la fonction érectile de l'llIEF dans les limites de la normale. II existait également dans ce groupe une amélioration significative de l'afflux artériel aux corps caverneux évalué par Doppler (vitesse systolique maximale $38 \pm 10 \mathrm{~cm} / \mathrm{sec}$ contre $29 \pm 9,8$ avant traitement, $p<0.05)$, tandis que le groupe "sildénafil à la demande" avait une amélioration discrète mais non significative, et que le groupe non traité présentait une discrète dégradation des résultats Doppler. De plus, des études Doppler faites six mois après l'arrêt de tout traitement chez les patients avec prise vespérale continue de sildénafil ont confirmé le maintien du bénéfice. Ces données préliminaires suggèrent que le traitement par administration chronique d'un inhibiteur de la PDE-5 peut induire des modifications tant structurelles que fonctionnelles du pénis susceptibles de guérir la DE. Ceci incite à approfondir les études de la fonction endothéliale du pénis.

Si les 3 inhibiteurs de la PDE- 5 ne sont disponibles sur le marché américain que depuis moins de 6 mois, nous en disposons en Europe depuis plus de 18 mois. Des ètudes de type "monde réel ", non sponsorisées par les compagnies pharmaceutiques, cherchant à vérifier l'efficacité, la sécurité d'emploi et la préférence des patients pour l'un ou l'autre inhibiteur apparaissent aujourd'hui et suscitent beaucoup d'intérêt.

Dans une étude multicentrique consacrée à des sujets avec DE n'ayant jamais pris jusque là d'inhibiteur de la PDE-5, 237 hommes ont reçu de façon randomisée des cures de six semaines de chaque inhibiteur aux doses recommandées (sildénafil $100 \mathrm{mg}$, tadalafil $20 \mathrm{mg}$, et vardénafil $20 \mathrm{mg}$ ), et une période équivalente de traitement placebo, tandis que 211 autres hommes ont reçu des doses inférieures de moitié aux précédentes (respectivement 50,10 et $10 \mathrm{mg}$ ) [5]. Chaque patient a donc reçu chacun des 3 inhibiteurs et le placebo, avec une semaine de washout entre deux types de traitement. Les principaux critères d'évaluation ont été l'augmentation des scores des questions Q3 (pénétration vaginale) et Q4 (maintien de l'èrection), les différents domaines de l'IIEF, la Global Assessment Question, et les scores obtenus à une échelle de préférence des patients.

Lors de la soumission de l'abstract, une analyse partielle des données montrait des gradients moyen d'augmentation des scores de la question Q3 de 0,3 pour le placebo, 1,5 pour le sildénafil, 1,5 pour le tadalafil, et 1,6 pour le vardénafil $(p<0.001)$. Les augmentations correspondantes pour la question Q4 étaient 0,5 pour le placebo, 1,7 pour le sildénafil, 1,6 pour le tadalafil, et 1,9 pour le vardénafil ( $p<$ 0.001 ). La préférence des patients dans le groupe avec administration de la dose maximale allait pour $43 \%$ au vardénafil, pour $17 \%$ au sildénafil, et pour $40 \%$ au tadalafil. Dans le groupe demi-dose, les proportions étaient respectivement de $50 \%$ pour le vardénafil, $31 \%$ pour le sildénafil et $19 \%$ pour le tadalafil. J'ajouterai personnellement (J.B.) que les données finales qui ont été présentées pour cette étude à San Francisco n'ont pas montré de supériorité nette de l'un des 3 inhibiteurs de la PDE- 5 en termes de puissance, de tolérance ou de préférence du patient, en notant que le schéma d'administration recommandé au patient était dans tous les cas un schéma de prise à la demande classique, une heure avant le rapport sexuel, ce qui, selon plusieurs intervenants dans la discussion, ainsi que de ma propre expérience, n'est pas celui qui met le plus en valeur le Tadalafil. 
Une autre étude allemande a cherché à déterminer la préférence pour l'un des trois inhibiteurs de la PDE- 5 après essai des trois médicaments selon un schéma ouvert [6]. Deux cent vint deux patients avec DE de durée moyenne 6,3 ans, âgés en moyenne de 57,9 ans, ont reçu chacun des trois inhibiteurs selon une séquence arbitraire. Tous les patients avaient subi un bilan extensif comportant l'IIEF, une étude Doppler des artères péniennes, des modules de préférence, etc. Les taux de préférence d'ensemble ont été de $44 \%$ pour le tadalafil, $32 \%$ pour le vardénafil, et $14 \%$ pour le sildénafil. Dix pour cent des hommes n'ont exprimé de préférence pour aucune des trois molécules. Parmi les 39 diabétiques, les taux de préférence étaient de $35 \%$ pour le vardénafil, $27 \%$ pour le tadalafil, $14 \%$ pour le sildénafil, et aucune préférence pour $24 \%$. En ce qui concerne les raisons pour la préférence de l'un ou l'autre agent, $96 \%$ des sujets ayant exprimé une préférence pour le Tadalafil l'ont imputée à la durée de son action, $61 \%$ de ceux qui préféraient le Vardénafil l'ont imputée à un début d'action plus rapide, et $77 \%$ de ceux qui ont préféré le Sildénafil ont cité une efficacité meilleure.

J'ajouterais personnellement (J.B.) qu'au cours de la discussion, l'auteur principal a précisé qu'il avait fait des recommandations différentes en fonction du type de molécule utilisée : prise à la demande une heure avant le rapport pour le sildénafil et le vardénafil, prise au moins 3 heures avant le rapport, et sans tenir compte de l'heure du repas pour le tadalafil. C'est à ce schéma particulier de prescription qu'il attribuait le taux de préférence plus élevé pour le Tadalafil dans l'ensemble de ses patients. Ceci rejoignait l'expérience de plusieurs autres spécialistes, exprimée au cours de la discussion qui a suivi. Cette étude comportait par ailleurs un défaut méthodologique important, dans la mesure où la majorité des patients n'étaient pas naïs, mais au contraire déjà sous sildénafil au moment de l'inclusion dans l'étude. Dans ce cas l'ordre d'administration des 3 médicaments ne pouvait pas être randomisé, et la période sildénafil était évaluée de façon rétrospective, pendant les dernières semaines du traitement en cours, ce qui introduisait un premier biais. De plus on peut penser que de ce fait d'avoir inclus des patients déjà sous traitement a pu sélectionner une population comportant une proportion plus élevée de sujets non satisfaits du sildénafil, puisque la majorité des patients satisfaits fait renouveler ses ordonnances par son médecin traitant, plutôt que d'aller consulter un spécialiste de haut niveau, à honoraires probablement conséquents du fait de sa pratique privée.

\section{Prothèses péniennes}

L'implantation de prothèses péniennes gonflables (PPG) chez les hommes pour qui les traitements moins invasifs ont échoué continue de donner des résultats satisfaisants à beaucoup d'hommes, spécialement aux Etats-Unis. Des innovations dans la conception et les matériels utilisés ont permis d'augmenter leur longévité. Cependant, le risque d'infection de la prothèse reste une préoccupation majeure pour les patients et les chirurgiens. Elle survient dans 1 à
$3 \%$ des cas après une première implantation, et dans 10 à $18 \%$ après révision chirurgicale. Les principaux fabricants de prothèses aux Etats-Unis (Mentor et American Medical Systems) ont introduit de nouveaux produits visant à diminuer le risque d'infection prothétique.

Les implants AMS $700 \mathrm{CX}$ sont imprégnés de minocycline et de rifampicine de façon à lutter contre les staphylocoques, le germe pathogène le plus commun en cas d'infection prothétique. Une présentation a été consacrée au bilan des révisions chirurgicales de prothèses survenues entre Mai 2001 et Septembre 2003 pour les prothèses " inhibizone " et " non-inhibizone " [7]. Parmi 8754 cas de révision chirurgicale effectuées après des durées d'implantation de 4 à 28 mois, 5310 réimplantations avaient utilisé des matériels non-inhibizone (non imprégnés d'antibiotique : $60,7 \%$ ) et 1344 , des implants inhibizones, $(39,9 \%)$. Le taux d'infection qui était de $2,4 \%$ dans les cas de révision non-inhibizone a été réduit à $1,36 \%$ avec les implants de type inhibizone (diminution de $44 \%$ ).

La prothèse pénienne gonflable Mentor Titan est recouverte de polyvinylpyrrolidone (PVP), une substance hydrophile qui réduit l'adhérence des bactéries et absorbe, puis diffuse, les antibiotiques dans lesquels la prothèse est immergée avant l'intervention. D'octobre 2000 à Août 2003, 2357 prothèses Titan recouvertes de PVP ont été implantées, et les résultats ont été comparés à ceux de l'implantation de 482 prothèses non recouvertes de PVP [8]. Le taux d'infection a été de $1,06 \%$ après prothèse recouverte de PVP contre $2,07 \%$ après prothèse non recouverte $(p<0.03)$. Les germes les plus souvent trouvés à la culture dans les deux groupes étaient des staphylocoques.

Il faudra manifestement disposer de plus de recul avant que ces procédés anti-infectieux puissent être considérés comme le traitement standard. On peut cependant penser que ce type de progrès permettra un jour ou l'autre d'améliorer et la satisfaction des patients et les résultats d'ensemble des prothèses péniennes.

Le caractère aisé de l'approche transcrotale pour l'implantation des prothèses péniennes et la proximité de l'urètre ont suggéré une stratégie combinée d'implantation des sphincters urinaires artificiels et des prothèses péniennes. Cependant la crainte d'augmenter le taux d'infection et par là d'aboutir à des résultats médiocres a limité l'acceptation des implantations simultanées. Une évaluation multi-institutionnelle de leurs résultats chez 22 patients implantés entre 2000 et 2003 a révélé deux érosions urétrales et une migration de réservoir, mais pas d'infection post-opératoire [9]. Les facteurs de risque incluaient un diabète chez sept sujets $(32 \%)$, une hypertension chez six $(27 \%)$ et des antécédents de radiothérapie chez six $(27 \%)$. Le taux de révision a été de $14 \%$ pour l'ensemble des cas. Tous les patients ont rapporté qu'ils avaient eu besoin de moins d'une couche par jour pour leurs fuites urinaires. Les patients à haut risque, du fait de problèmes urologiques complexes, ont un risque de complications plus élevé. L'avantage de l'anesthésie unique et de l'incision transcrotale unique devrait inciter à recourir plus largement à cette technique. 


\section{Hypertrophie bénigne de la prostate (HBP), troubles de l'érection et troubles de l'éjaculation}

Lors du dernier meeting de l'AUA, à Chicago, la Multinational Survey on Aging Males (MSAM)-7 a démontré que les symptômes du bas appareil urinaire (LUTS) sont un facteur de risque indépendant pour les dysfonctions sexuelles, tant $D E$ que dysfonction éjaculatoire (DEj), qui sont toutes deux fréquentes et perturbantes chez les hommes âgés. Suite à cette étude, l'Asian Survey on Aging Males (ASAM) a tenté de déterminer la prévalence du LUTS et des problèmes sexuels chez les hommes asiatiques âgés, et d'étudier la relation entre LUTS et dysfonction sexuelle dans cette population [10]. Cette évaluation a été conduite dans sept pays asiatiques.

L'étude a évalué les données démographiques, les symptômes urinaires (questionnaires IPSS et Quality of Life Index), les aspects fonctionnels (questionnaires DANPSSsex et IIEF) et les facteurs de co-morbidité. Mille cent cinquante cinq hommes âgés de 50 à 80 ans ont complété l'enquête. II s'est trouvé confirmé que la prévalence et la sévérité des LUTS étaient fortement corrélés à l'âge. Une proportion importante des sujets évalués (72\%) étaient encore actifs sexuellement. Dans l'ensemble, la DE était fréquente $(63 \%)$, mais la DEj l'était encore plus $(68 \%)$. Cependant, la gêne causée par ces dysfonctions sexuelles chez les Asiatiques était faible, ce qui est nettement différent de ce qu'on a observé chez les Américains. Dans l'ensemble, ces résultats confirment une relation entre LUTS et dysfonction sexuelle.

En ce qui concerne les problèmes d'éjaculation, l'éjaculation prématurée (EP) est l'une des dysfonctions sexuelles masculines les plus courantes, avec une prévalence dépassant $30 \%$. Elle est pour sa plus grande part méconnue et non traitée, en partie du fait stigmate lié au terme EP. Le groupe de travail de la Société de Médecine Sexuelle a organisé un débat pour discuter de l'éventuelle nécessité de renommer cette dysfonction éjaculation rapide ou précoce [11]. Après avoir écouté de multiples arguments provenant d'experts en matière de problèmes sexuels, des rapports du Comité des Recommandations de I'AUA, de patients participants à la réunion, de groupes de discussion, et de représentants des professions de santé, le groupe de travail a conclu que changer le nom d'éjaculation prématurée engendrerait une confusion, induirait la nécessité d'un travail d'information considérable, et retarderait les efforts qui doivent être faits pour augmenter la prise de conscience de ce problème par le public. La recommandation finale a été de continuer d'utiliser le terme d'éjaculation prématurée, et de développer des stratégies pour réduire la connotation péjorative associée à ce problème, et pour améliorer l'information et la communication à propos de l'EP.

\section{Substitution hormonale}

Une étude a utilisé les sujets de la Massachussetts Male Aging Study (MMAS) pour évaluer la prévalence et l'incidence du déficit androgénique lié à l'âge dans une popula- tion représentative d'hommes d'âge moyen ou élevé [12]. Lors de la première évaluation, les prévalences des déficits androgéniques possible et certain étaient de 5,4 et de 1,6 $\%$. Lors d'une réévaluation 8 à 10 ans plus tard, les taux étaient de 12,2 et 2,3\%. Si l'on tient compte de ces chiffres, il y aurait aux Etats-Unis presque trois millions d'hommes âgés de 40 à 70 ans qui auraient un déficit androgénique possible ou certain.

\section{Recherche fondamentale}

Une quantité d'excellentes études de recherche fondamentale ont été rapportées, étudiant les aspects moléculaires de la fonction érectile et les traitements potentiels des DE à partir de modèles animaux expérimentaux. II y a également eu des papiers représentatifs consacrés aux populations difficiles à traiter que ce soit du fait de l'âge, de délabrements nerveux après prostatectomie radicale, ou du diabète.

Une des découvertes récentes les plus importantes en matière de recherche biomédicale est que des cellules souches peuvent être trouvées dans de nombreux tissus de l'adulte, et peuvent fournir de nouvelles cellules pour refaire un tissu normal, étant donc susceptibles de régénérer des tissus endommagés ou malades. Les cellules stromales de la moelle osseuse (MSCs), encore appelées cellules souches mésenchymateuses, sont des cellules souches adultes qu'on trouve dans la moelle osseuse et qui ont un potentiel de différentiation multilinéaire, contribuant à la régénération des tissus mésenchymateux tels que l'os, le cartilage, la graisse et le muscle. Puisqu'il est relativement facile d'isoler ces MSCs, de les développer in vitro, et de manipuler leurs gènes, des MSCs génétiquement modifiées ont été utilisées récemment pour libérer des gènes et régénérer des tissus pour traiter différentes maladies. Dans une étude consacrée à la DE liée au vieillissement, des chercheurs ont décrit une méthode de transfert dans le pénis des rats âgés de NOS endothéliale via le transfert de MSCs de rats modifiées génétiquement, dans la perspective d'une application potentielle de ce type de thérapie génique au traitement des DE [13].

Les résultats démontrent que les MSCs de rat, seules ou potentialisées génétiquement par la NOS endothéliale, peuvent améliorer l'érection altérée des rats âgés. II est important de noter que l'administration des MSCs de rat a été à l'origine d'une augmentation des cellules musculaires lisses et endothéliales, qui étaient actives et exprimaient une quantité considérable de NOS endothéliale dans les corps caverneux, contribuant par conséquent à améliorer la fonction des corps caverneux et donc la fonction érectile. Ces données suggèrent que cette nouvelle variété de thérapie génique, basée sur l'administration de cellules, peut être utile pour restaurer la fonction des cellules endothéliales et musculaires lisses du pénis, et donc la fonction érectile.

Avec l'arrivée de nouveaux vecteurs utilisables pour la thérapie génique, cette nouvelle approche thérapeutique des dysfonctions érectiles est devenue une réalité au cours des 
cinq dernières années. L'utilisation de l'approche génétique dans le but de restaurer le signal neuronal au niveau des nerfs endommagés par une prostatectomie radicale constitue une option intéressante pour les DE de cette catégorie de patients. Des chercheurs allemands ont ainsi étudié les effets d'un apport de cellules de Schwann modifiées par un facteur neurotrophique dérivé d'une lignée cellulaire gliale (GDNF) aux nerfs caverneux après qu'ils aient excisé une partie de ces nerfs de façon bilatérale chez des rats [14].

Leurs résultats montrent que les animaux qui avaient subi la résection nerveuse avaient des perturbations significatives de la fonction érectile en réponse à la stimulation des nerfs caverneux. Cependant, les rats qui avaient subi cette résection des nerfs caverneux, mais qui avaient aussi reçu des greffes de cellules de Schwann hyper-secrétant le GDNF avaient par rapport aux rats témoins une amélioration significative de leur fonction érectile et une régénération axonale avec de nouvelles fibres de type B et de type C. Ces données suggèrent donc que la greffe de cellules de Schwann hyper-secrétant le GDNF sous l'effet d'une thérapie génique pourrait être utilisée pour traiter les DE post-prostatectomie radicale, du fait de la capacité de ces cellules à stimuler la régénération des fibres nerveuses.

Les problèmes d'érection des diabétiques résultent de nombreux mécanismes, incluant dysfonction endothéliale, neuropathie, stress oxydatif, et modifications structurelles du lit vasculaire du pénis. De telles altérations de la vascularisation périphérique peuvent expliquer la prévalence élevée $(>50 \%)$ de la $D E$ chez les diabétiques. Dans la $D E$ diabétique, l'un des facteurs contributifs majeurs est la réduction du nombre des fibres nerveuses contenant de la NO synthase, à l'origine d'une diminution de la production de NO, et d'une altération de la relaxation des fibres musculaires lisses induite par les mécanismes endothéliaux et neurologiques. Récemment, la voie RhoA/Rho-kinase, qui influence le tonus du muscle lisse, a été décrite. On a montré que cette nouvelle cascade de signaux inhibe la NO synthase endothéliale, et diminue donc le NO d'origine endothéliale dans le lit vasculaire systémique. Une étude récente vient d'apporter les premières preuves, à la fois au niveau moléculaire et au niveau fonctionnel, d'une régulation de l'expression de la NO synthase endothéliale dans les corps caverneux des diabétiques par la voie RhoA/Rho-kinase [15].

Ces données suggèrent la possibilité d'un mécanisme par lequel les diabétiques peuvent développer une DE consécutive à la diminution de la production de NO résultant d'une augmentation de l'activité de la voie de signalisation RhoA/Rho-kinase au niveau des vaisseaux caverneux. L'inhibition de la voie RhoA/Rho-kinase par transfert viral au pénis de rats diabétiques d'un gène mutant dominant inhibant la RhoA augmente l'expression pénienne de la NO synthase endothéliale, augmente l'activité NO synthase constitutive, et les taux de GMP cyclique, et par là-même restaure la vasodilatation et la fonction érectile dépendantes de la fonction endothéliale. Par conséquent la voie RhoA/Rho-kinase peut constituer une nouvelle cible thérapeutique pour le traitement de la DE diabétique.

\section{Divers}

Le rôle joué par les médecines alternatives dans le traitement de nombreuses pathologies continue d'augmenter. Les inhibiteurs de la PDE-5 se sont imposés d'eux-mêmes comme traitement oral de premier choix pour le traitement des DE. II n'est pas étonnant qu'on ait vanté certains produits d'origine végétale en affirmant qu'ils avaient la même efficacité que les inhibiteurs de la PDE-5, et avec en plus l'avantage d'être des traitements "naturels ". Dans une étude récente, une analyse en chromatographie liquide à haute performance a été pratiquée sur 7 produits dont on prétendait qu'ils amélioraient la qualité de l'érection. On a trouvé la présence d'inhibiteurs de la PDE-5 à concentration significative dans 2 de ces 7 produits d'origine prétendument végétale [16].

Consommateurs et organismes de contrôle doivent en être avertis car les inhibiteurs de la PDE-5 peuvent avoir des interactions graves, sinon mortelles, lorsqu'on les associe à d'autres médicaments, par exemple les dérivés nitrés. Le public doit être informé que le terme " naturel " n'implique pas nécessairement sécurité. Ces préparations devraient faire l'objet d'une véritable régulation.

De même, de nombreux patients recueillent des informations sur la DE sur internet. Par l'intermédiaire des liens internet, un grand nombre de traitements d'origine végétale est proposé à la vente, et des recommandations pratiques sont faites. Une étude anglaise a revu plus de 160000 hits sur des remèdes végétaux proposés pour la $\mathrm{DE}$. $80 \%$ des sites correspondants soulignaient l'efficacité de ces médicaments, $70 \%$ les fournissaient, et seulement $21 \%$ précisaient leurs effets indésirables et leurs contre-indications [17]. Seuls $21 \%$ des sites correspondants disposaient d'un personnel médical supervisant les informations, et seuls $18 \%$ présentaient une information référencée.

Les ingrédients les plus souvent proposés ou décrits étaient la yohimbine, le ginseng et le gingko biloba. Bien que des effets indésirables graves aient été rapportés avec ces produits (par exemple des manifestations hémorragiques et des arythmies cardiaques avec le gingko biloba), la plupart des sites internet déclaraient que ces remèdes végétaux étaient sans danger. Les résultats de cette étude démontrent à l'évidence les dangers qu'il y a pour les consommateurs à acheter des traitements d'origine végétale pour la DE sur des sites internet non contrôlés.

\section{Conclusions}

Une nouvelle fois l'AUA 2004 a permis la présentation de nombreux travaux dans le domaine de la sexualité et de ses problèmes, particulièrement en ce qui concerne les études moléculaires et la thérapie génique, l'éjaculation prématurée, les problèmes sexuels féminins, et la maladie de La Peyronie. Les études moléculaires sont encore à leur tout début, et on s'attend à des développements futurs en ce qui concerne les agents à action centrale, les traitements transcutanés et la thérapie génique. 


\section{REFERENCES}

1. GIULIANO F., ZERBIB M., ROSEN R. : Erectile dysfunction (ED) in urological practice settings : prevalence, severity according to the IIEF-5 and distress in 1303 patients. J. Urol., 2004 : 171, 871A.

2. MICHL U., GRAEFEN M., PALISAAR J. et al. : Erection, potency, and sexual intercourse following unilateral nerve sparing prostatectomy with $n$.suralis transplantation contralateral compared to nerve sparing prostatectomy : a critical analysis of preoperative potent men. J. Urol., $2004: 171,890 \mathrm{~A}$.

3. LEVINE L., Mc CULLOUGH A., PADMA-NATHAN H. : Longitudinal randomized placebo-controlled study of the return of nocturnal erections after nerve-sparing radical prostatectomy in men treated with nightly sildenafil citrate. J. Urol., 2004 : $171,875 \mathrm{~A}$.

4. SOMMER F., ENGELMANN U. : What are the long term effects on erectile dysfunction of taking sildenafil on a daily basis? J. Urol., $2004: 171,903 \mathrm{~A}$

5. SOMMER F., KLOTZ T., MATHERS M., BONDARENKO B., CASPERS $H$., ENGELMANN U. : Which PDE-5 inhibitor do patients prefer ? A comparative randomized placebo-controlled multicenter study. J. Urol., 2004 : 171, 1192A.

6. PORST H., KLEINGARN $M$ : The three PDE-5 inhibitors sildenafil, tadalafil, and vardenafil -- results of a comparative preference trial in 222 patients with erectile dysfunction. J. Urol., $2004: 171,1193 \mathrm{~A}$.

7. CARSON C. : Initial success with AMS 700 series inflatable penile prosthesis with inhibizone antibiotic surface treatment : a retrospective review of revision cases : incidence and comparative results versus non-treated devices. J. Urol., 2004 : $171,894 \mathrm{~A}$.

8. WOLTER C., RAJPURKAR A., KENDIRCI M., DHABUWALA C., HELLSTROM W. : Hydrophilic-coated inflatable penile prosthesis : one-year experience. J. Urol., 2004 : 171, 896A

9. SHAW K., MOREY A., JONES L., HAKIM L., SHANNON S., HELLSTROM W. : Synchronous prosthetic implantation through a trans-scrotal incision: an outcome analysis. J. Urol., $2004: 171,898$ A.

10. LI M., ROSEN R. : LUTS and male sexual dysfunction in Asia. J. Urol., $2004: 171,873 \mathrm{~A}$

11. BARADA J., LEWIS R., WAGNER G. : Should the condition of premature ejaculation be renamed ? J. Urol., $2004: 171$, $881 \mathrm{~A}$.

12. ARAUJO A., O'DONNELL A., BRAMBILLA D., SIMSON W., MCKINLAY J. : Prevalence and incidence of androgen deficiency in middle-aged and older men : estimates from the Massachussets Male Aging Study. J. Urol., 2004 : 171 $1616 \mathrm{~A}$.

13. BIVALACQUA T., USTA M., CHAMPION H., DENG W., KADOWITZ P., HELLSTROM W. : Mesenchymal stem cells alone or modified with eNOS restore erectile function in the aged rat : cell-based gene therapy for erectile dysfunction. J. Urol., 2004 : 171, 1417A.

14. MAY F., WEIDNER N., MATIASEK K., et al. : Bridging the gap: GDNF-hypersecreting Schwann cell grafts restore erectile function after ablation of cavernous nerve segments. $J$. Urol., 2004 : 171, 1425A.
15. BIVALACQUA T., USTA M., CHAMPION H. et al. : RhoARhokinase suppresses endothelial nitric oxide synthase in the penis : novel mechanism for diabetic-associated erectile dysfunction. J. Urol., 2004 : 171, 1411A.

16. FLESHNER N., HARVEY M., ADOMAT H., WOOD C., HERSEY K., GUNS E. : Evidence for pharmacological contamination of herbal erectile function products with type 5 phosphodiesterase (PDE-5) inhibitors. J. Urol., 2004 : 171, 1190A.

17. THURAIRAJA R., BARRASS B., PERSAD R. : Obtaining herbal remedies for erectile dysfunction from the internet is a potentially dangerous sexual practice. J. Urol., $2004: 171$, $1191 \mathrm{~A}$. 